\title{
DETECTION OF HARD EXUDATES USING SIMULATED ANNEALING BASED THRESHOLDING MECHANISM IN DIGITAL RETINAL FUNDUS IMAGE
}

\author{
Diptoneel Kayal $^{1}$ and Sreeparna Banerjee ${ }^{2}$ \\ ${ }^{1,2}$ School of Computer Science \& Engineering, West Bengal University of \\ Technology, Kolkata, India \\ ${ }^{1}$ diptoneel@gmail.com, ${ }^{2}$ sreeparnab@hotmail.com
}

\begin{abstract}
Diabetic retinopathy is a disease commonly found in case of diabetes mellitus patients. It causes severe damage to retina and may lead to complete or partial visual loss. In case of diabetic retinopathy retinal blood vessel gets damaged and protein and fat based particles gets leaked out of the damaged blood vessels and are deposited in the intra-retinal space. They are normally seen as whitish marks of various shape and are called as exudates. Exudates are primary indication of diabetic retinopathy. As changes occurs due to the disease is irreversible in nature, the disease must be detected in early stages to prevent visual loss. But detection of exudates in early stages of the disease is extremely difficult only by visual inspection because of small diameter of human eye. But an efficient automated computerized system can have the ability to detect the disease in very early stage. In this paper we have proposed one such method.
\end{abstract}

\section{KEYWORDS}

Diabetic retinopathy, exudates, median filtering, thresholding, simulated annealing.

\section{INTRODUCTION}

Diabetic retinopathy is one of the most serious complications of diabetes mellitus and a major cause of blindness. It is a progressive disease classified according to the presence of various clinical abnormalities. It is the most common cause of blindness among people aged 30-69 years [1]. One-fifth of patients of diabetes type II, have retinopathy at the time of diagnosis. In type I diabetes, diabetic retinopathy never occurs after diagnosis. But after 15 years all most of all patients with type I and two-third of those with type II diabetes have background of diabetic retinopathy [1]. In case of diabetic retinopathy blood vessels get damaged and protein and fat based particles gets leaked out of the damaged blood vessels beside blood flow to the retina decreases. These particles are referred to as exudates. Various methods have been developed for detection of exudates. These include thresholding and edge detection based techniques [2], FCM based approach [3], gray level variation based approach [4], multilayer perceptron based approach [5]. Normalized cut image segmentation based method for detection of hard exudates has also been proposed by us [6]. Optic disk must be detected and segmented early in the detection 
process, as often optic disk has more or less same brightness and contrast as the exudates. So, if optic disk is not segmented in early stage the process may produce wrong result.

The proposed algorithm accepts input image in RGB format. As grayscale image comprises of mby-n image matrix, whereas RGB image comprises of m-by-n-by-3 image matrix and it is easier to operate on an m-by-n matrix than on m-by-n-by-3 grayscale image is used for whole process. If the input is in RGB then the input image is converted into a grayscale image for the ease of operation.

\section{Proposed Method}

The proposed method has five steps.

- Median filtering

- Image subtraction

- Application of simulated annealing

- Thresholding

- Image addition

\subsection{Median Filtering}

Digital image noise usually appears in the high frequency of the image spectrum. So, a low-pass digital filter may be used for noise removal. Linear low-pass filters tend to smear image details, whose power is in high frequencies as well. They also tend to blur image edges, thus degrading image quality. But a non-linear low-pass filter can remove noise while preserving the edges. Such a filter is based on data ordering. Let $x i, i=1,2, \ldots . n$ be $n$ observations, whose number $n=2 v+1$ is odd, can be ordered according to their magnitude as follows:

$$
\mathrm{x}(1)<\mathrm{x}(2)<\ldots \ldots \ldots .<\mathrm{x}(\mathrm{n})
$$

xi denoted i-th order statistic. $x(1), x(n)$ are the maximum and minimum observations respectively. The observation $\mathrm{x}(\mathrm{v}+1)$ lies in the middle and is called median of the observations. It is also denoted by med(xi). By definition, the median lies in the middle of the observation data. It minimizes the L1 norm:

$$
\sum_{i=1}^{n}\left|x_{i}-\operatorname{med}\right| \rightarrow \min
$$

According to (2) the median is the maximum likelihood estimate of the location for the Laplacian distribution:

$$
f(x)=1 / 2 \exp (-|x|)
$$

A two-dimensional median filter has the following definition

$$
y(i, j)=\operatorname{med}\{x(i+r, j+s), \quad(r, s) \in A \quad(i, j) \in Z 2\}
$$

Where $\mathrm{Z} 2=\mathrm{Z} \times \mathrm{Z}$ denotes the digital image plane. The set $\mathrm{A} \subset \mathrm{Z} 2$ defines the filter window. If the input image is of finite extent $\mathrm{N} \times \mathrm{M}, 0 \leq \mathrm{i} \leq \mathrm{N}-1,0 \leq \mathrm{j} \leq \mathrm{M}-1$, definition (4) is valid only in the interior of the output image, that is, for those $i, j$ for which 


$$
0 \leq \mathrm{i}+\mathrm{r} \leq \mathrm{N}-1,0 \leq \mathrm{j}+\mathrm{s} \leq \mathrm{M}-1,(\mathrm{r}, \mathrm{s}) \in \mathrm{A}
$$

(5) is not valid at the border of the image. There are two approaches solve this problem. In the first one, the filter window A is truncated in such a way so that (5) is valid and definition (4) can be used again. In the second approach, the input sequence is appended with sufficient samples and (4) is applied for

$$
0 \leq \mathrm{i} \leq \mathrm{N}-1,0 \leq \mathrm{j} \leq \mathrm{M}-1
$$

Median filter can remove additive white noise. They are very efficient in the removal of noise having long-tailed distribution. The median is a robust estimator of location also. Therefore a single outlier (e.g. impulse) can have no effect on its performance, even if its magnitude is very large or small. The median becomes unreliable only if more than $50 \%$ of the data are outlier. The robustness of median filter makes it very suitable for impulse noise filtering. This property of median filter can be used for this approach. As in the original input image exudates are bright areas with comparatively high contrast than that of its neighboring region if we apply median filter on the input image (in grayscale format) we would obtain a filtered image in which the exudates are blurred to a great extent. Beside as the optic disk part has almost same brightness as that of exudates this part of retinal fundus image will also becomes blurred. Median filter has another interesting property of preserving sharpness of edges of the image. Preservation of edge information and its enhancement is a very important subjective feature of the performance of digital image filter. Median filters not only smoothes noise in homogenous image regions but tends to produce regions of constant intensity. All these properties make median filter ideal for this approach.

\subsection{Image Subtraction}

Next step of this approach is subtraction of median filtered image from input image (in case of the input image is in grayscale form) or subtraction of median filtered image from grayscale form on input image (in case of the input image is in RGB form). Image subtraction is used to find changes between two images of same scene. Mathematically image subtraction can be denoted as follows:

$$
c(m, n)=f(m, n)-g(m, n)
$$

As mentioned earlier median filtering makes the brighter regions (i.e. exudates) into blur, hence the result of subtraction gives us the output in which only regions with high brightness and contrast can be observed. Subtraction is one of the most important step of this process as in this step the desired features of input retinal fundus image is extracted.

\subsection{Application of Simulated Annealing}

Simulation annealing is a well known probabilistic metaheuristic technique for finding global optima in a large search space. It was first proposed by Kirkpatric, Gelett, Vecchi (1983) [7] and Cerny (1985) [8]. In this method every point (S) of search space is analogous to a state of some physical system. At each step simulated annealing considers some neighbouring state s' of the current state s and probabilistically decides whether to move to state s'. This method is repeated until the system reach to an optimal point. The algorithm normally employs a random search which not only accept changes that decrease the objective function (f) but also accept the changes the increase the objective function with a probability given by

$$
\mathrm{p}=\exp (-\delta \mathrm{f} / \mathrm{T})
$$


where $\delta \mathrm{f}$ is the increase in $\mathrm{f}$ and $\mathrm{T}$ is control parameter. Most important advantage of simulated annealing over other methods is that it's ability to avoid becoming trapped in local minima. Another useful advantage is that it does not rely on any restrictive properties which increases it's versatility. In our proposed method we have used simulated annealing to determine the value of thresholding parameter. In order to do this we have considered the value of each pixel after subtraction step. The values of the pixels provides us the search space upon which we have applied simulated annealing to get the optimum values of the search space and that value is used as the value of thresholding parameter in next step of our method.

\subsection{Thresholding}

If in an image consists of light objects on a dark background, in such a way that object and background pixels have intensity values grouped into dominant modes, then we can extract light objects from background using thresholding operation. Then any point $(\mathrm{x}, \mathrm{y})$ in the image at which $\mathrm{f}(\mathrm{x}, \mathrm{y})>\mathrm{T}$ is called an object point, where $\mathrm{T}$ is known as threshold parameter. Otherwise the point is called a background point. Thresholding operation can be defined as follows:

$$
g(x, y)= \begin{cases}1 & \text { if } f(x, y)>T \\ 0 & \text { if } f(x, y) \leq T\end{cases}
$$

\subsection{Image Addition}

Image addition is used to create double exposure. If $\mathrm{f}(\mathrm{m}, \mathrm{n})$ and $\mathrm{g}(\mathrm{m}, \mathrm{n})$ represents two images then addition of these two images to get the resultant image is given by

$$
c(m, n)=f(m, n)+g(m, n)
$$

If multiple images of a given region are available for approximately the same data and if a part of one of the image has some noise then the part can be compensated from other images available through image addition.

\section{RESULT}

The proposed algorithm is implemented MATLAB 7.0.4. Configuration of the computers used for development and testing purpose is Intel Core2Duo $1.5 \mathrm{GHz}$ processor and $1 \mathrm{~GB}$ of RAM. The algorithm is tested on a database of ten images, among which seven images with exudates and three images with no exudates. All the images are collected from diaretdb0 database and the images are selected randomly.The ground data is verified by an expert ophthalmologist. For evaluation purpose value of True Positive (TP), False Positive (FP) and False Negative (FN) parameters are determined for each image. Sensitivity $(\mathrm{S})$ and Predictivity $(\mathrm{P})$ is used for the measurement of accuracy. Sensitivity and predictivity are defined as follows.

Sensitivity $(\mathrm{S})=\mathrm{TP} /(\mathrm{TP}+\mathrm{FN})$ and Predictivity $(\mathrm{P})=\mathrm{TP} /(\mathrm{TP}+\mathrm{FP})$

The overall sensitivity and predictivity are found to be $98.66 \%$ and $98.12 \%$ respectively. 
Table 1

\begin{tabular}{|c|c|c|c|c|c|}
\hline & TP & FP & FN & $\mathbf{S}=\frac{\boldsymbol{T P}}{\boldsymbol{T P}+\boldsymbol{F N}}$ & $\mathbf{P}=\frac{\boldsymbol{T P}}{\boldsymbol{T P}+\boldsymbol{F P}}$ \\
& & & & & 100 \\
\hline IMAGE 1 & 5 & 0 & 0 & 100 & 100 \\
\hline IMAGE 2 & 8 & 0 & 0 & 100 & 100 \\
\hline IMAGE 3 & 7 & 0 & 0 & 100 & 100 \\
\hline IMAGE 4 & 11 & 0 & 2 & 100 & 100 \\
\hline IMAGE 5 & 14 & 0 & 2 & 100 & 1.25 \\
\hline IMAGE 6 & 13 & 2 & 3 & 86.60 & 100 \\
\hline IMAGE 7 & 4 & 0 & 0 & 100 & 100 \\
\hline IMAGE 8 & 0 & 0 & 0 & 100 & 100 \\
\hline IMAGE 9 & 0 & 0 & 0 & 100 & $\mathbf{9 8 . 1 2 \%}$ \\
\hline IMAGE 10 & 0 & 0 & 0 & 100 & \\
\hline OVERALL & & & $\mathbf{9 8 . 6 6 \%}$ & \\
\hline
\end{tabular}

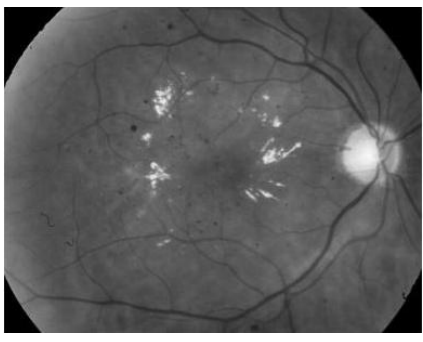

Figure 1. Input image

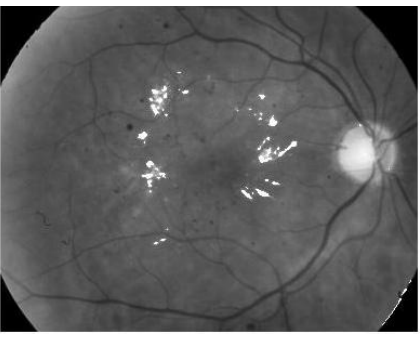

Figure 2. Output image

\section{REFERENCES}

[1] R. Klein, B. Klein, S. Moss, M. Davis, D. Demants, “ The Wisconsin epidemiology study of diabetic retinopathy type II", Archieve of Opthalmology, Vol 102, No 4, pp 520-526, 1984

[2] A. V. Sagar, B. Balasubramaniam, V. Chandrasekhara, " A Novel Intergrated Approach Using Dynamic Thresholding and Edge Detection for Automatic Detection of Exudates in Digital Fundus Retinal images”, IEEE International Conference on Computing, 2007, Page 286-292.

[3] H. Li, O. Chutatape, “A Model Based Approach for Automated Feature Extraction in fundus Images', IEEE International conference on Computer Vision, 2003, Page 127-133

[4] A. Osrach, B. Shadgar, R. Markmham, "A Computational Intelligence Based Approach for Detection of Exudates in Diabetic Retinopathy", IEEE 2009

[5] T. Walter, J. C. Klein, P. Massin, A. Erginay, "A contribution of Image Processing To the Diagnosis of Diabetic Retinopathy - Detection of Exudates in Color Fundus Image of Human Retina", IEEE Transaction on Medical Imaging, Vol 21, No 10, October 2002, Page 256-264.

[6] D. Kayal, S. Banerjee, "An Approach to Detect Hard Exudates Using Normalized Cut Image Segmentation Technique in digital Retinal Fundus Image", First International Conference on Signal Processing, Image Processing and Pattern Recognition, 2012, New Delhi, India.

[7] S. Kirkpatric, C. D. Gelett, M. P. Vecchi, "Optimization by simulated annealing”, Science, pp621630, 1983.

[8] V. Cerny, "A thermodynamic approach to the travelling salesman problem : An efficient simulation" Journal of Optimizaton Theory and application, pp 41-51, 1985.

[9] M. Garcia, R. Hornero, C. I. Sanchez, M. I. Lopez, A. Diez, "Feature Extraction and Selection for Automatic Detection of Hard Exudates in Retinal Images", conference of the IEEE EMBS, France, 2007. 
[10] C. Sinthanayothin, V. kongbunkiat, S. Phoojaruenchanachai, A. Singalavanija "Automated Screening System For Diabetic Retinopathy", $3^{\text {rd }}$ international Symposium On Image And Signal Processing And Analysis, 2003, Page 915-920

[11] Wareham NJ, "Cost Effectiveness of Alternative Methods for Diabetic Retinopathy Screening" Diabetes Care, 2003, Vol 16, Page 844

[12] S. Ravishankar, A. Jain, A. Mittal, "Automated Feature Extraction for Early Detection of Diabetic Retinopathy in Fundus Images”, IEEE 2009, Page 210-218

[13] Z. Liu, C. Opas, S. Krishnan, "Automatic Image Analysis for Fundus Image" $19^{\text {th }}$ International Conference of IEEE EMBS, Chicago 1997, Page 524-528

[14] D. Kayal, S. Banerjee, "A Method To Detect Hard Exudates Using Normalized Cut Image Segmentation Technique in Digital Retinal Fundus Image”, First International Conference on Signal Processing, Image Processing and Pattern Recognition 2012, New Delhi, India.

\section{Authors}

\section{Diptoneel Kayal}

Diptoneel Kayal obtained his B.E. in I.T. and M.Tech in Software Engineering, both from West Bengal University of Technology. He is presently working as a Ph.D scholar at West Bengal University of Technology. His research interest includes medical image processing and cloud computing

\section{Dr. Sreeparna Banerjee}

Dr Sreeparna Banerjee obtained her B. Sc., M. Sc., and Ph.D degrees all in Physics. She has taught in universities in India and abroad. Her current research interests include Physics of space plasmas: Molecular Dynamics and Monte Carlo simulations,charge transfer, nonlinear dynamics; Neural networks, Pattern Recognition and Soft Computing applications in Astrophysics, Meteorology and Medical Imaging; Data Mining.
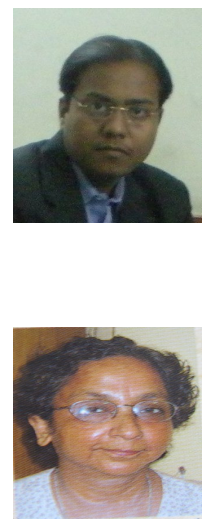